\title{
Propagation Path Loss Modeling and Outdoor Coverage Measurements Review in Millimeter Wave Bands for 5G Cellular Communications
}

\author{
Mohammed B. Majed1, Tharek A. Rahman², Omar Abdul Aziz ${ }^{3}$ \\ ${ }^{1,2,3}$ Wireless Communication Center (WCC), Faculty of Electrical Eng., Universiti Teknologi Malaysia (UTM), \\ Johor, Malaysia \\ ${ }^{1}$ Department of Computer Science, College of Science and Technology, University of Human Development (UHD), \\ Assulaymaniyah, Iraq
}

\begin{abstract}
Article Info
Article history:

Received Jan 19, 2018

Revised Mar 29, 2018

Accepted Apr 14, 2018

Keyword:

$5 \mathrm{G}$

Mm-wave

PDPs

Propagation path loss

RMS delay spread

ABSTRACT

The global bandwidth inadequacy facing wireless carriers has motivated the exploration of the underutilized millimeter wave (mm-wave) frequency spectrum for future broadband cellular communication networks, and mmWave band is one of the promising candidates due to wide spectrum. This paper presents propagation path loss and outdoor coverage and link budget measurements for frequencies above $6 \mathrm{GHz}$ (mm-wave bands) using directional horn antennas at the transmitter and omnidirectional antennas at the receiver. This work presents measurements showing the propagation time delay spread and path loss as a function of separation distance for different frequencies and antenna pointing angles for many types of real-world environments. The data presented here show that at $28 \mathrm{GHz}, 38 \mathrm{GHz}$ and $60 \mathrm{GHz}$, unobstructed Line of Site (LOS) channels obey free space propagation path loss while non-LOS (NLOS) channels have large multipath delay spreads and can utilize many different pointing angles to provide propagation links. At $60 \mathrm{GHz}$, there is more path loss and smaller delay spreads. Power delay profiles PDPs were measured at every individual pointing angle for each TX and RX location, and integrating each of the PDPs to obtain received power as a function of pointing angle. The result shows that the mean RMS delay spread varies between $7.2 \mathrm{~ns}$ and $74.4 \mathrm{~ns}$ for $60 \mathrm{GHz}$ and $28 \mathrm{GHz}$ respectively in NLOS scenario.
\end{abstract}

Copyright $@ 2018$ Institute of Advanced Engineering and Science. All rights reserved.

\section{Corresponding Author:}

Mohammed B. Majed,

Wireless Communication Center (WCC),

Faculty of Electrical Eng.,

Universiti Teknologi Malaysia (UTM),

Johor. Malaysia.

Email: mohammed.majed@uhd.edu.iq

\section{INTRODUCTION}

Wireless communications technology has been developed fast and frequently to provide the requirements for the modern techniques in different applications. However, the high data rate and fast communication demand increases more and more [1]. In the year 2020, wireless data traffic is expected to increase by 1000 fold and may increase by 10,000 fold by 2025 [2]. For cellular communication, the cellular capacity must be increased to face the growing traffic demand.

Today there are a lot of multimedia services arises with the evolution of the mobile devices industry and rapid development in the mobile communication sector and the using of mobile communication at these days does not depend on voice communication only, it includes also broadband and multimedia services that 
the mobile communication infrastructure can support, but on the first place is always a user demand for high mobility, high data rate and high availability [3]. All these user requirements make the mobile communication industry searching for a new technology and new frequency spectrum to support their infrastructure to meet the user requirements [4]. The experiences of current mobile and wireless communications networks have shown that data traffic, especially, is growing more than anticipated. This development is providing a significant challenge to the development of future mobile and wireless communication networks [5]. It is envisioned that future IMT systems, in addition to other features, will need to support very high throughput data links to cope with the growth of the data traffic [6].

International mobile telecommunications (IMT)-advanced specifications of fourth generation (4G) terrestrial mobile telecommunication were approved by the international telecommunication union radio standards sector (ITU-R) in January 2012. Meanwhile, the dramatic growth of mobile data services driven by wireless Internet and smart devices has triggered the investigation of $5 \mathrm{G}$ for the next generation of terrestrial mobile telecommunications [7]. 5G wireless networks are expected to be a mixture of network tiers of different sizes, transmit powers, backhaul connections, different radio access technologies (RATs) that are accessed by an unprecedented numbers of smart and heterogeneous wireless devices [8]. This architectural enhancement along with the advanced physical communications technology such as high-order spatial multiplexing multiple-input multiple-output (MIMO) communications will provide higher aggregate capacity for more simultaneous users, or higher level spectral efficiency, when compared to the 4G networks [9].

\section{RADIO PROPAGATION MODEL}

A radio propagation model is an empirical mathematical formulation for the characterization of radio wave propagation as a function of frequency, distance and other characteristics. A single model is usually developed to predict the behavior of propagation for every similar link under similar constraints. The essential aim of signal propagation is to formalize how the signal can propagate from one point to another. Only in such situation can a typical model predict the path loss effect on an area covered by a single or multi transmitter (s) [10]. In wireless communications, radio propagation between base station and terminals is affected by such mechanisms as scattering, diffraction and reflection [11].

The radio coverage is determined by radio signal path loss, which increases with increasing frequency. The RF power of radio signals would be reduced when radio signals have travelled over a considerable distance. Therefore, in most cases, the systems with higher frequencies will not operate reliably over the distances required for the coverage areas with varied terrain characteristics [12]. For clear line of sight (LOS) propagation, the range between the transmitter and receiver is determined by the free space path loss equation, given by:

$$
\text { Panthloss }=[20 \log 10] \frac{4 \pi d}{\lambda} d B
$$

where $d$ and $\lambda$ are the range and wavelength in meters, respectively.

In Non-Line-of-Sight (NLOS) cases, the performance of higher frequencies is worse with reliable distances dropping even faster. Most paths are obstructed by objects and buildings. When penetrating obstacles, radio waves are decrease in amplitude. As the radio frequency increases, the rate of attenuation increases. Figure 1 illustrates the effect of higher frequencies having higher attenuation on penetrating obstacles [1].

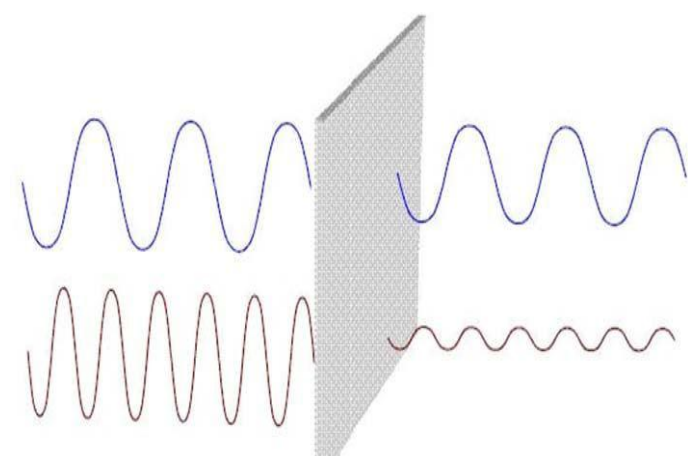

Figure 1. Higher frequencies have higher attenuation on penetrating obstacles 
A radio beam can diffract when it hits the edge of an object. The angle of diffraction is higher as the frequency decreases. When a radio signal is reflected, some of the RF power is absorbed by the obstacle, attenuating the strength of the reflected signal. Figure 2 show that higher frequencies lose more signal strength on reflection [4].
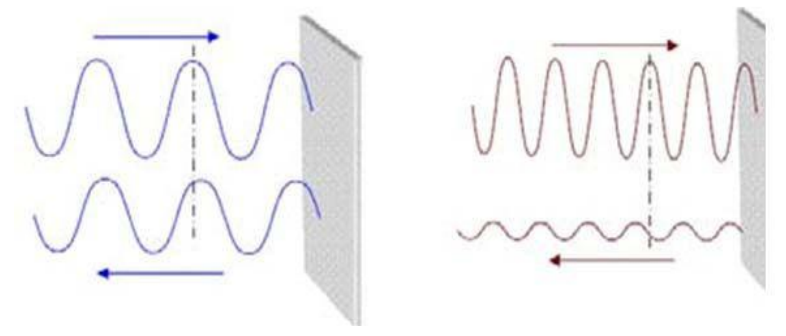

Figure 2. Frequency dependence of signal strength on reflection

In free space propagation, clear and unobstructed line-of-sight (LOS) path is available and the first Fresnel zone is maintained between base station and terminal. Free space path loss can be obtained by using the logarithmic value of the ratio between the receiving and transmitting power. Equation (2) indicates that free space path loss is frequency dependent and it increases with distance. The increase of distance and frequency produce similar effect on the path loss.

$$
P L_{d B}=92.44+20 \log 10 f_{G H z}+20 \log d_{K m}
$$

where $f$ is frequency, $d$ is distance respectively.

Figure 3 shows the free space path loss at frequencies above $6 \mathrm{GHz}$ for different ranges.

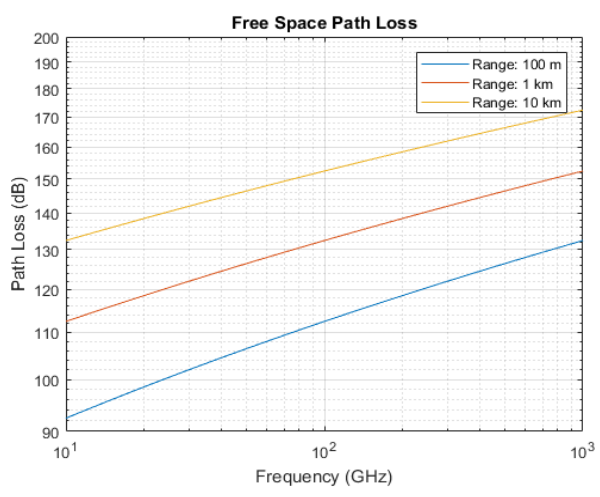

Figure 3. Free space path loss at frequencies above $6 \mathrm{GHz}$ for different ranges

\section{PROPAGATION PATH LOSS MEASUREMENTS}

One of the difficulties of mobile communications in the higher bands for outside get to can't avoid being to beat the normal troubles in spread conditions. The most clear obstruction will be the higher way loss of the groups over $6 \mathrm{GHz}$ in respect to conventional cell groups [13], [14]. Utilizing a free-space reference of 3 meters, tests in urban micro cell outdoor-to-outdoor scenarios, with transmitter and receiver antenna heights below rooftop, measured path loss exponents for $10 \mathrm{GHz}, 28 \mathrm{GHz}, 38 \mathrm{GHz}$, and $60 \mathrm{GHz}$ in both LOS and NLOS situations in distance $200 \mathrm{~m}$, which are abridged in Table 1 below.

Table 1. Path Loss Exponents Measured in Several Frequencies

\begin{tabular}{ccccc}
\hline Frequency & $10 \mathrm{GHz}$ & $28 \mathrm{GHz}$ & $38 \mathrm{GHz}$ & $60 \mathrm{GHz}$ \\
\hline NLOS & 3.4 & 3.5 & 4.3 & 3.76 \\
LOS & 2 & 2.1 & 3.1 & 2.25 \\
\hline
\end{tabular}


For comparison, Table 2 contrasts the deliberate LOS and the NLOS way misfortune got from the $10 \mathrm{GHz}$ and $28 \mathrm{GHz}$ path loss exponents in the urban micro cell outdoor-to-outdoor experiments as well as $38 \mathrm{GHz}$ and $60 \mathrm{GHz}$. The values are registered for different small cell pertinent separations. The free-space path loss (FSPL) model is considered, FSPL reference remove demonstrate, provides a path loss exponent which has physical importance since the way misfortune is attached to the FSPL at a particular close-in reference separate $(1 \mathrm{~m}$ is helpful and down to earth at millimetric wave frequencies). Figure 4 demonstrates that the deliberate directional LOS and NLOS path loss is near the free-space path loss with an exponent of 2 in both the backhaul and access cases for 28 and $38 \mathrm{GHz}$.

Table 2. Path Loss Correlation for LOS and NLOS Situations in different Frequencies

\begin{tabular}{cccccccccccccc}
\hline Frequency & & \multicolumn{3}{c}{$10 \mathrm{GHz}$} & \multicolumn{3}{c}{$28 \mathrm{GHz}$} & \multicolumn{3}{c}{$38 \mathrm{GHz}$} \\
\hline Distance & Meters & 20 & 100 & 200 & 20 & 100 & 200 & 20 & 100 & 200 & 20 & 100 & 200 \\
NLOSPath Loss & $\mathrm{dB}$ & 91.3 & 113.5 & 124 & 95.6 & 121.7 & 132.3 & 103.9 & 131.1 & 142.1 & 107.6 & 135.8 & 147.4 \\
LOS Path Loss & $\mathrm{dB}$ & 79.4 & 91.8 & 97.9 & 84.2 & 96.3 & 104.7 & 91 & 104.2 & 109.8 & 97.3 & 112.7 & 119.3 \\
\hline
\end{tabular}

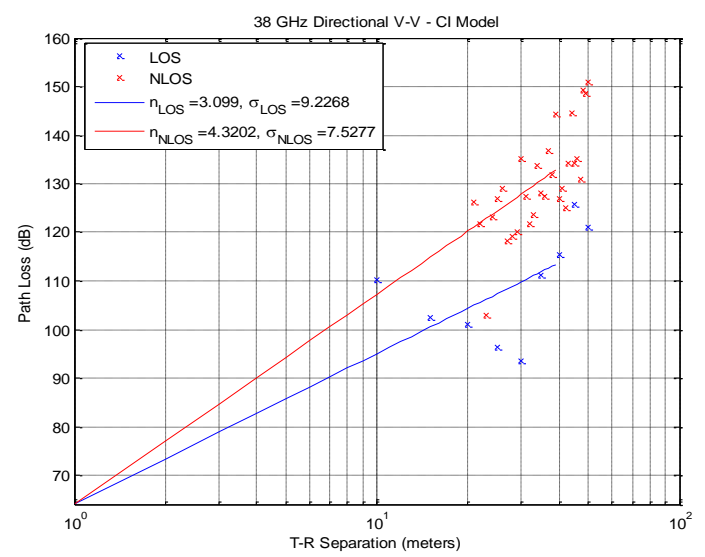

Figure 4. Measured directional antenna path loss calculated with respect to $1 \mathrm{~m}$ free-space path loss for $38 \mathrm{GHz}$

The directional path loss models were produced by considering the deliberated power delay profiles PDPs at each individual directing plot for every TX and RX area, also, coordinating each of the PDPs to acquire received power as a component of guiding edge, and after that subtracting the TX and RX antenna gains from each individual power estimation. At each increasing stride along the range in the azimuth plane, a PDP was recorded at the receiver. Figure 5 and Figure 6 show the measurements in different frequencies.

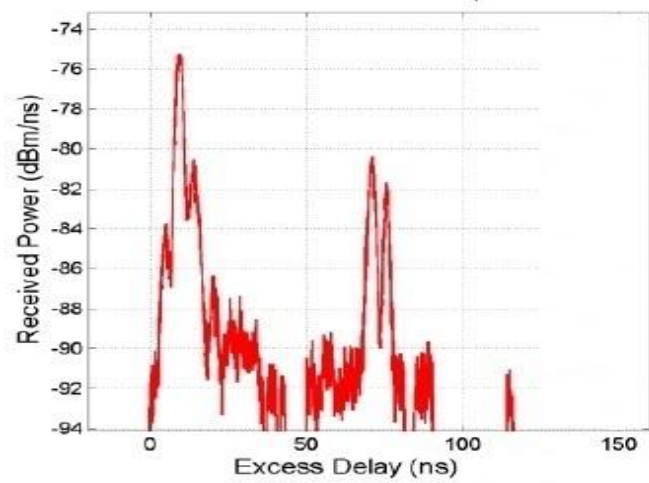

Figure 5. PDPs Measured at $28 \mathrm{GHz}$

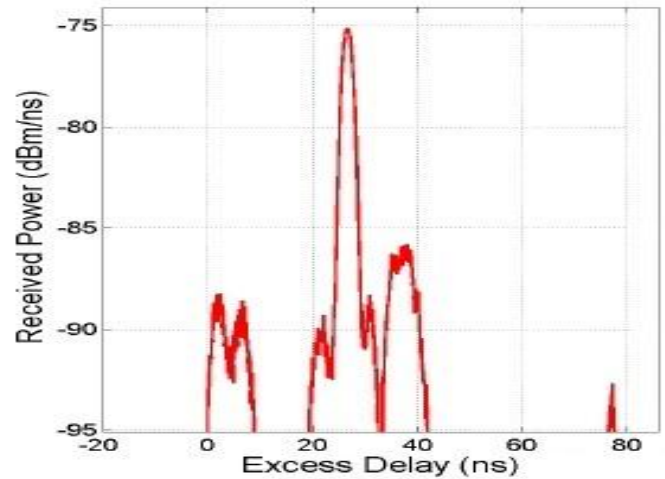

Figure 6. PDPs Measured at $60 \mathrm{GHz}$ 


\section{RMS DELAY SPREAD MEASUREMENTS}

Root-Mean-Square (RMS) delay spread is expanded for lower gain antennas which utilize more extensive beams, as the more extensive profile gathers signals from more headings with comparative or equivalent gain to the boresight point. This especially applies to user equipment UE whose size and power prerequisites do not bolster considerable arrays and have a more omni-directional pattern as shown in Figure 7.

Then again, RMS delay spread is diminished for higher gain antennas and the related smaller beamwidth. The transmit beamwidth from the base station restricts the bearing of the created vitality and subsequently the chances to disperse. Moreover, despite the higher gain, scattered vitality of the multipath connection may not be grabbed by the spatial range of the receive antenna boresight.

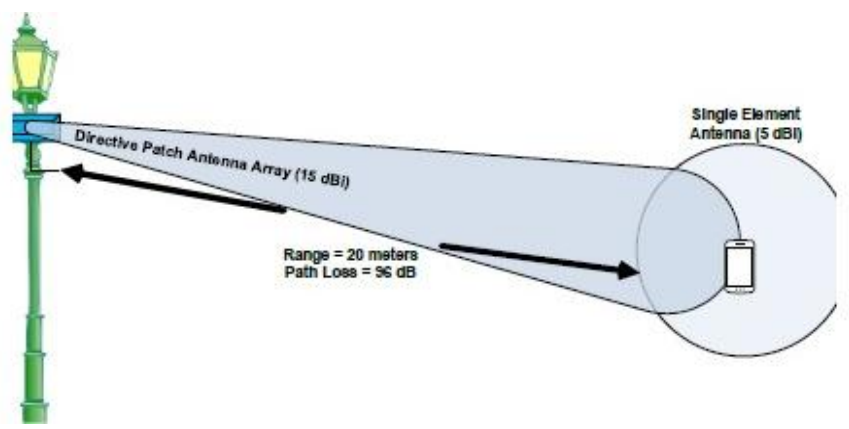

Figure 7. Link budget scenario

A transmitter beamwidth of 6 degrees and the transmitter separation of 100 meters, the UE recipient will be lit up by the essential transmitter vitality and its reflections over a circular segment length of around 10 meters. The reflections will hence be essentially limited by deferrals around $31 \mathrm{~ns}$. In the interim, higherarrange beams (i.e., beams with more reflections) have bigger angles of incidence, thus, more inclined to fall outside of the receiver antenna beamwidth. Hypothetically, for a run of the mill geometry of lampposts a few meters over the ground and few hundred meters separate, second request frameworks are regularly considered adequate approximations.

Along these lines, for a given situation and utilize cases with various transmitter and receiver antenna radiation patterns, one may watch diverse scattering effects as represented, in a somewhat perfect sense for simplicity of conceptualization, in Figure 8. The essential point is that delay spread is moderated by the beamforming model. The analysis ledat millimetric wave frequencies in outdoor environments. This experiment included NLOS scenario over an assortment of a few frequencies. The findings are condensed in Table 3.

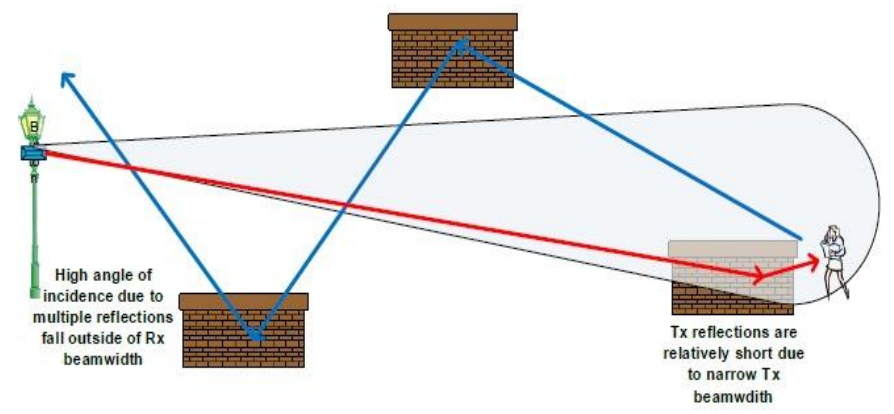

Figure 8. Scattering effects

Table 3. Outline of Channel RMS Delay Spread for NLOS exp

\begin{tabular}{lccc}
\hline & $28 \mathrm{GHz}$ & $38 \mathrm{GHz}$ & $60 \mathrm{GHz}$ \\
\hline Mean RMS delay spread & $74.4 \mathrm{~ns}$ & $22.8 \mathrm{~ns}$ & $7.2 \mathrm{~ns}$ \\
Max RMS delay spread & $455.3 \mathrm{~ns}$ & $184.1 \mathrm{~ns}$ & $37.7 \mathrm{~ns}$ \\
\hline
\end{tabular}




\section{OUTDOOR-TO-OUTDOOR COVERAGE AND LINK BUDGET}

The primary thought for link budget analysis is the signal power attenuation because of spread wastage over the air. Free space path loss FSPL additionally increments in extent to the square of the separation between the transmitter and receiver. As such, a $28 \mathrm{GHz}$ signal transmitted over a distance of 25 meters loses $90 \mathrm{~dB}$ of power simply covering this generally short separation between transmitter and receiver. At 100 meters, the loss is expanded to $104 \mathrm{~dB}$. Coverage can be analyzed from the link budget point of view. Since the typical outdoor urban environments will incorporate NLOS ways, the investigation ought to incorporate the NLOS cases. For the given system parameters of Table 4, the extreme distances that can support 1 Gbps data rate in different situations can be found in this section.

Table 4. System Parameters for Link Budget Analysis

\begin{tabular}{cccc}
\hline Carrier Frequency $(\mathrm{GHz})$ & 28 & 38 & 60 \\
Tx EIRP + Rx Gain (dBm) & 66 & 68 & 69 \\
Bandwidth (GHz) & 1 & 0.5 & 2 \\
Rx Noise Figure (dB) & 6 & 9 & 9 \\
Other losses (dB) & 10 & 10 & 10 \\
Target SNR (dB) & 0 & N/A & N/A \\
Target Data Rate (Gbps) & 1 & 1 & 1 \\
\hline
\end{tabular}

In the analysis displayed in Table 5 , the $28 \mathrm{GHz}$ frequency band is considered for the center frequency of systems with $1 \mathrm{GHz}$ bandwidth. Tx EIRP and Rx gain are assumed to be $66 \mathrm{dBm}$, which can be realized by low-power base stations. $30 \mathrm{dBm}$ Tx power with $26 \mathrm{dBi}$ Tx antenna gain and $10 \mathrm{dBi} \mathrm{Rx}$ antenna gain have been used for the systems. As appeared in the Table 5, the low-power base station can give 1 Gbps using $1 \mathrm{GHz}$ bandwidth for the outdoor coverage with from tens to hundreds meter cell range contingent upon cell situations.

Table 5. Link Budget Analysis for different Environments at $28 \mathrm{GHZ}$

\begin{tabular}{cccc}
\hline Environments & Open Space & Campus & Dense Urban \\
\hline LoS / NLoS & LoS & NLoS & NLoS \\
Path loss model & PL $(\mathrm{d})=61.4+$ & PL $(\mathrm{d})=47.2+$ & PL $(\mathrm{d})=61.4+$ \\
Max. distance for 1 Gbps & $20 * \log 10(\mathrm{~d})$ & $29.8 * \log 10(\mathrm{~d})$ & $34.1 * \log 10(\mathrm{~d})$ \\
\hline
\end{tabular}

\section{CONCLUSION}

A propagation path loss, RMS delay spread, and outdoor-to-outdoor coverage measurements at a range of frequencies above $6 \mathrm{GHz}$ (up to $60 \mathrm{GHz}$ ) in LOS and NLOS scenarios have been analyzed. The specific frequencies used in the measurements $(10 \mathrm{GHz}, 28 \mathrm{GHz}, 38 \mathrm{GHz}$, and $60 \mathrm{GHz})$ are arbitrarily selected and intended to illustrate the general trends of how coverage varies across the frequency range.

Outdoor studies conducted at different frequencies showed that consistent coverage can be achieved by having base stations with a cell-radius of 200 meters. Path loss was in NLOS and higher frequencies larger than in LOS and lower frequencies. Multipath delay spread is found to be much larger in lower frequencies $(28 \mathrm{GHz})$ due to small coherence bandwidth. The key trends include near free-space path loss and virtually no RMS delay spread for all LOS links, while NLOS links have higher RMS delay spread, as much as $455.3 \mathrm{~ns}$ (for the $28 \mathrm{GHz}$ ) and $37.7 \mathrm{~ns}$ (for the $60 \mathrm{GHz}$ ). In general, NLOS links offer increasing RMS delay spread as the azimuth pointing angles are increased away from boresight at either or both the transmitter and receiver. By picking the best combination of transmitter and receiver antenna pointing angles at any location, path loss and RMS delay spread can be reduced substantially.

Some short-range communication technologies, like millimeter -wave communication technology, can be seen as promising candidates to provide high quality, bandwidth required for mobile broadband applications and high data rate services to outdoor and indoor users. And we have analyzed the suitability of different mmwave frequencies for mobile communication.

\section{REFERENCES}

[1] J. G. Andrews, et al., "What Will 5G Be?" IEEE J. Sel. Areas Commun., vol. 32, no. 6, pp. 1065-1082, 2014.

[2] M. Cudak, et al., "Moving towards mmwave-based Beyond-4G (B-4G) technology," in IEEE Vehicular Technology Conference, 2013.

[3] T. S. Rappaport, “The massively broadband future," Spectrum 20/20 conference, 2012. 
[4] S. Chen and J. Zhao, "The requirements, challenges, and technologies for 5G of terrestrial mobile telecommunication," IEEE Communications Magazine, vol. 52, no. 5, pp. 36-43, 2014.

[5] Gareh M., et al., "Path Loss Models Optimization for Mobile Communication in Different Areas," Indonesian Journal of Electrical Engineering and Computer Science, vol. 3, no. 1, pp. 126-35, 2016.

[6] A. Maltsev, et al., "MmWave Smallcells is a Key Technology for Future 5G Wireless Communication Systems," European Conference on Networks and Communications (EuCNC'2014), Bologna, Italy, 2014.

[7] M. R. Akdeniz, et al., "Millimeter Wave Channel Modeling and Cellular Capacity Evaluation," IEEE Journal on Selected Areas in Communications, Special Issue on 5G, 2014.

[8] Hossain, et al., "Evolution toward 5G multi-tier cellular wireless networks: An interference management perspective," IEEE Wireless Communications, vol. 21, no. 3, pp. 118-127, 2014.

[9] Wang, et al., "Cellular architecture and key technologies for 5G wireless communication networks," IEEE Communications Magazine, vol. 52, no. 2, pp. 122-130, 2014.

[10] A. Maltsev, et al., "WP5: Propagation, Antennas and Multi-Antenna Techniques," MiWEBA EU, 2014.

[11] Israr I., et al., "Path Loss Modeling of WLAN and WiMAX Systems," International Journal of Electrical and Computer Engineering, vol. 5, no. 5, pp. 1, 2015.

[12] Recommendation ITU-R P.452-12, "Prediction Procedure for the Evaluation of Microwave Interference between Stations on the Surface of the Earth at Frequencies above about $0.7 \mathrm{GHz}$," Geneva, Switzerland, 2007.

[13] Rasekh, et al., "A street canyon approximation model for the $60 \mathrm{GHz}$ propagation channel in an urban environment with rough surfaces," in Telecommunications (IST), 2010 5th International Symposium on, pp. 132-137, 2010.

[14] Esmail B. A., et al., "Reconfigurable Metamaterial Structure at Millimeter Wave Frequency Range," International Journal of Electrical and Computer Engineering (IJECE), vol. 7, no. 6, pp. 2942-9, 2017 